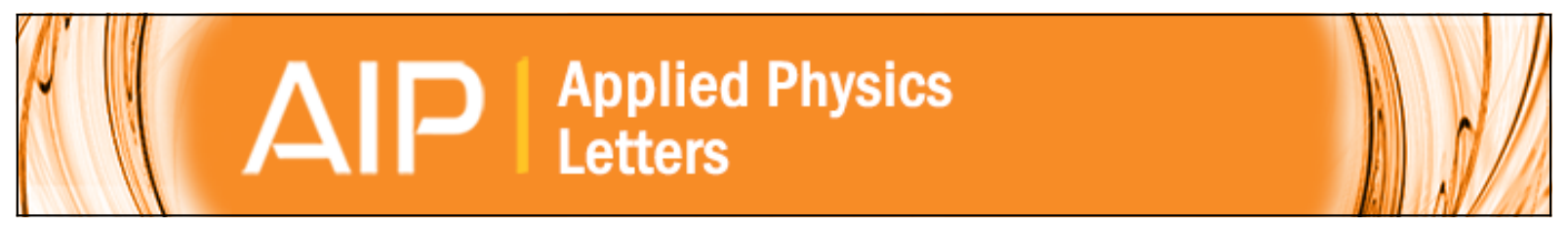

Simultaneous current-, force-, and work-function measurement with atomic resolution

M. Herz, Ch. Schiller, F. J. Giessibl, and J. Mannhart

Citation: Applied Physics Letters 86, 153101 (2005); doi: 10.1063/1.1900316

View online: http://dx.doi.org/10.1063/1.1900316

View Table of Contents: http://scitation.aip.org/content/aip/journal/apl/86/15?ver=pdfcov

Published by the AIP Publishing

Articles you may be interested in

A method to measure the thermovoltage with a high spatial resolution

Appl. Phys. Lett. 108, 141601 (2016); 10.1063/1.4945665

Measurement of work function difference between $\mathrm{Pb} / \mathrm{Si}(111)$ and $\mathrm{Pb} / \mathrm{Ge} / \mathrm{Si}(111)$ by high-order Gundlach oscillation

J. Appl. Phys. 114, 214308 (2013); 10.1063/1.4839335

Simultaneous measurement of tunneling current and atomic dipole moment on $\mathrm{Si}(111)-(7 \times 7)$ surface by noncontact scanning nonlinear dielectric microscopy

J. Appl. Phys. 113, 014307 (2013); 10.1063/1.4772705

Simultaneous atomic force and scanning tunneling microscopy study of the Ge ( 111$)$ - c ( $2 \times 8$ ) surface J. Vac. Sci. Technol. B 28, C4D1 (2010); 10.1116/1.3305340

High-sensitivity quantitative Kelvin probe microscopy by noncontact ultra-high-vacuum atomic force microscopy Appl. Phys. Lett. 75, 286 (1999); 10.1063/1.124357

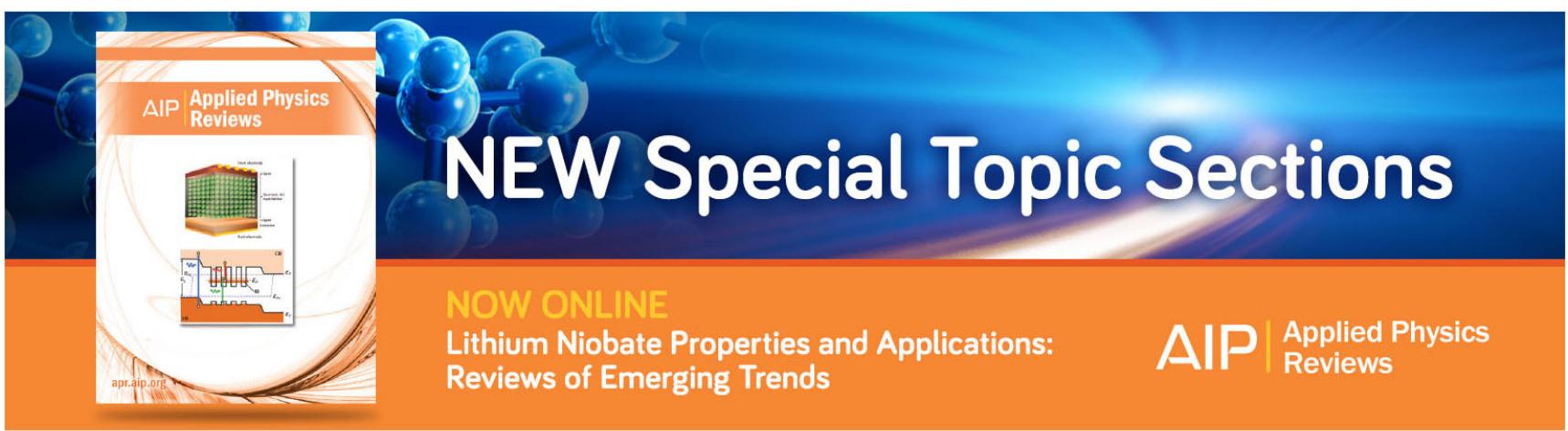




\title{
Simultaneous current-, force-, and work-function measurement with atomic resolution
}

\author{
M. Herz, Ch. Schiller, F. J. Giessibl, ${ }^{a)}$ and J. Mannhart \\ Universität Augsburg, Institute of Physics, Electronic Correlations and Magnetism, \\ Experimentalphysik VI, Universitätsstrasse 1, D-86135 Augsburg, Germany
}

(Received 4 October 2004; accepted 9 March 2005; published online 4 April 2005)

\begin{abstract}
The local work function of a surface determines the spatial decay of the charge density at the Fermi level normal to the surface. Here, we present a method that enables simultaneous measurements of local work-function and tip-sample forces. A combined dynamic scanning tunneling microscope and atomic force microscope is used to measure the tunneling current between an oscillating tip and the sample in real time as a function of the cantilever's deflection. Atomically resolved work-function measurements on a silicon $(111)-(7 \times 7)$ surface are presented and related to concurrently recorded tunneling current and force measurements. (C) 2005 American Institute of Physics.
\end{abstract}

[DOI: 10.1063/1.1900316]

When two metallic electrodes are separated by a small vacuum gap and a bias voltage $V_{t}$ is applied, a quantum mechanical tunneling current flows. ${ }^{1-3}$ For metallic electrodes, the tunneling current increases approximately by a factor of 10 for each distance reduction of $100 \mathrm{pm}$. This sharp distance dependence is key to the atomic resolution capability of the scanning tunneling microscope (STM). ${ }^{4}$ In one dimension, the decay of the tunneling current with distance is roughly given by

$$
I=I_{0} \exp (-2 \kappa z)
$$

with

$$
\kappa=\sqrt{2 m \Phi / \hbar},
$$

where $m$ is the mass of an electron and $\Phi$ is the local work function or barrier height. ${ }^{3}$ As Binnig et al. has shown early in the development of STM, this exponential dependence holds over at least $0.5 \mathrm{~nm}$ or so for tunneling currents ranging from a few hundred pA to a few $\mu \mathrm{A} .{ }^{2}$ Due to the atomic structure of matter, $\kappa$ is also a function of the lateral positions $x$ and $y$ as well as $z$ (see Fig. 1) for very small tunneling distances.

A straightforward method for measuring $\kappa(x, y, z)$ is to stop the lateral scan over a specific atom position and perform a $I(z)$ measurement. However, this method is time consuming and prone to errors from creep and drift of the piezoelectric scanner. Pethica et al. ${ }^{5}$ have extended "currentimaging-tunneling spectroscopy" by Hamers et al., ${ }^{6}$ an acmethod for current versus voltage spectroscopy where a small ac voltage is added to $V_{t}$ to recover the density of states. In the Pethica et al. method, the tip is oscillated at a fixed frequency $f$ (typically on the order of a few $\mathrm{kHz}$ ) according to $\vec{x}=\vec{x}_{0}+\vec{A} \cos (2 \pi f t) .^{5}$ For this situation, Eq. (1) needs to be generalized to

$$
I=I_{0} \exp (-2 \vec{\kappa} \vec{x}) .
$$

The natural logarithm of the normalized current is then given by

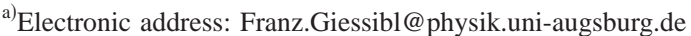

$$
\ln \left(I / I_{0}\right)=-2 \vec{\kappa} \vec{x}_{0}-2 \vec{\kappa} \vec{A} \cos (2 \pi f t) .
$$

In general, we define an apparent decay "constant" $\widetilde{\kappa}$ $=\eta /(2 A)$, where $\eta$ is the ac component of $\ln \left(I / I_{0}\right)$. For the case described by Eq. (4), $\eta=2 \vec{\kappa} \vec{A}$.

Atomic force microscopy (AFM) has progressed rapidly in the past years, ${ }^{7,8}$ and a combination with other techniques like $\mathrm{STM}^{9}$ or kelvin probe microscopy ${ }^{10}$ became feasible. Here, we combine AFM with the Pethica et al. method by using a qPlus sensor ${ }^{11}$ where the STM tip is mounted on a vibrating cantilever. The oscillation frequency is no longer fixed in this case, but varies by a frequency shift $\Delta f$ as determined by the atomic forces acting between tip and sample. The frequency shift data can then be related to forces, ${ }^{8}$ and simultaneous measurements of forces and decay constants are possible.

Because the atomically resolved STM image is influenced by the atomic and subatomic structure of the tip and sample wave functions, ${ }^{3,9,12}$ these structures also influence the decay constant images. ${ }^{5}$ The tunneling current can be calculated with a plane-wave expansion of the surface wave

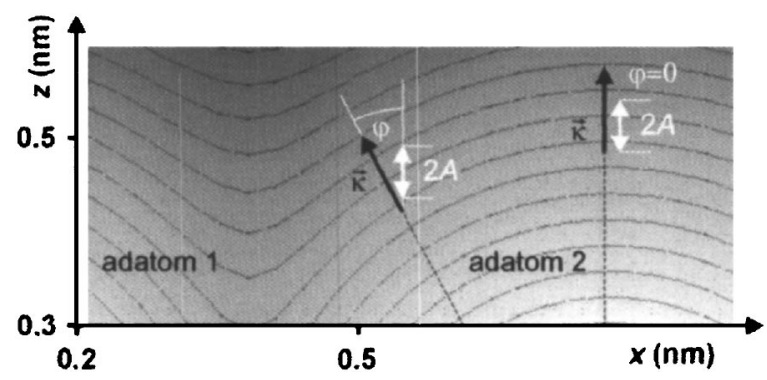

FIG. 1. Schematic view of $\ln \left(I / I_{0}\right)$ above two silicon adatoms located at $z$ $=0$ and $z=0.77 \mathrm{~nm}$ of a silicon $(111)-(7 \times 7)$ surface. The surface is oriented perpendicular to the $z$ axis. The current distribution between each of the silicon states and a $s$ tip is approximately given by $I \propto(z / r)^{2} \exp (-2 \vec{\kappa} \vec{r})$ (Ref. 3). The amplitude of the vertical oscillation is $A . \varphi$ is the angle between the oscillation direction and $\vec{\kappa}$. For decreasing $\cos (\varphi)$, the decay strength of the current in vertical direction also decreases (see text). Note that generally $\vec{\kappa}$ is not parallel to $\vec{\nabla} \ln \left(I / I_{0}\right)$ because of the angular factor of the wave function. The distance of the contour lines corresponds to a current increase of 1.82 . 

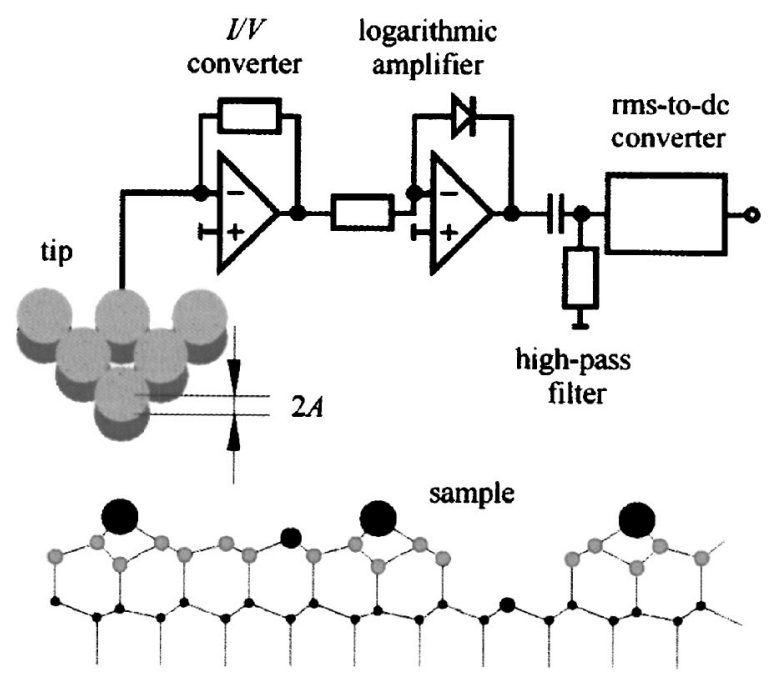

FIG. 2. Principle of dynamic work-function measurement. The tip is mounted on a quartz cantilever (not shown here, see Ref. 11 for details) with a stiffness of $1800 \mathrm{~N} / \mathrm{m}$ which oscillates at a fixed amplitude $A \approx 0.1 \mathrm{~nm}$. The unperturbed resonance frequency is $f_{0} \approx 20 \mathrm{kHz}$. Due to the sinusoidal oscillation of the cantilever, the tunneling current is strongly modulated at a frequency $f \approx f_{0}$ (see Fig. 3).

functions, ${ }^{3}$ ultimately depending on the superposition of atomic orbitals. ${ }^{3,9,12}$

For the atomic basis functions at the Fermi level, the exponential radial factor is $\exp (-\vec{\kappa} \vec{r})$, where $\vec{\kappa}=\kappa \vec{r} / r$ and $\vec{r}$ is the position vector with respect to the nucleus. When the tip moves by $\vec{\alpha}$, the corresponding conductivity distribution changes according to $\exp (-2 \vec{\kappa} \vec{\alpha}){ }^{3}$ Figure 1 shows a cross section of a model distribution of $\ln \left(I / I_{0}\right)$ for two atomic silicon states of a silicon $(111)-(7 \times 7)$ surface, with the nuclei on the $x$ axis at $z=0$, separated by $0.77 \mathrm{~nm}$. The current dependence $I \propto(z / r)^{2} \exp (-2 \vec{\kappa} \vec{r})$ is a good fit for tunneling between a tip ( $s$ tip) and each of the $s p^{3}$-adatom states. ${ }^{3}$ The tip is assumed to oscillate vertically. $\varphi$ is the angle between the oscillation direction and $\vec{\kappa}$. For $\cos (\varphi) \rightarrow 1, \eta$ approaches $2 \vec{\kappa} \vec{A}=2 \kappa A$ in this model. Therefore, an apparent barrier height $\Phi_{\text {app }}=\hbar^{2} \widetilde{\kappa}^{2} /(2 m)$ can be extracted from the value of $\widetilde{\kappa}$ at the position of the single atom. For decreasing $\cos (\varphi), \widetilde{\kappa}$ also decreases in the theoretical model, since $\vec{A}$ is no longer parallel to the direction of the fastest decay of the current. In the model, odd higher powers of $\left[\ln \left(I / I_{0}\right)\right](q)$ $=\left[\ln \left(I / I_{0}\right)\right]\left(\vec{x}_{0}+q \vec{A} / A\right)$ influence the value of $\widetilde{\kappa}$ as well.

In this article, we present dynamic STM/AFM measurements ${ }^{9}$ performed in ultrahigh vacuum at a pressure of $p \approx 10^{-8} \mathrm{~Pa}$ and ambient temperature $T \approx 300 \mathrm{~K}$. A silicon $(111)-(7 \times 7)$ surface is probed by a tungsten tip that is mounted on a qPlus force sensor. ${ }^{11}$ The qPlus sensor used here has a stiffness of $k=1800 \mathrm{~N} / \mathrm{m}$ and an eigenfrequency of $15487 \mathrm{~Hz}$. The oscillation amplitude of the cantilever needs to be approximately below $0.1 \mathrm{~nm}$ such that $I$ is above the noise level of the current amplifier even when the tip is at the far point of the surface. Operation at such small amplitudes poses a challenge as amplitude fluctuations tend to increase with decreasing amplitude, and only the use of cantilevers with a very large stiffness decreases amplitude fluctuations to an acceptable level. ${ }^{8}$ The experimental setup is shown in Fig. 2.

Figure 3 shows a simulation of the excursion of the tip mounted on a qPlus cantilever, the normalized tunneling cur-

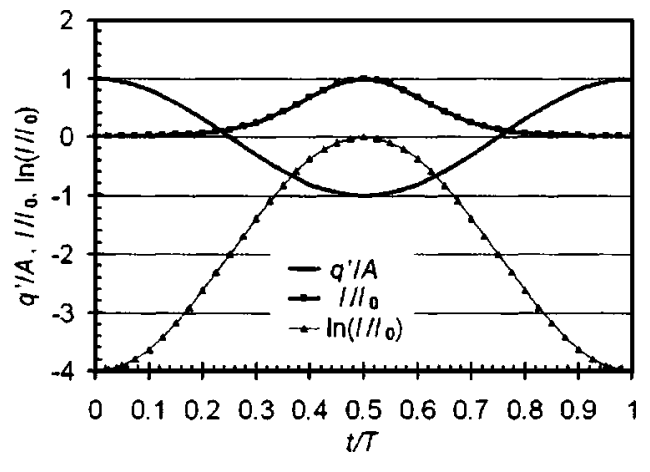

FIG. 3. Tip deflection divided by amplitude, current divided by maximum current and natural logarithm of $I / I_{0}$ as a function of time $t$ for $t=0$ to $t$ $=1 / f$. If the oscillation amplitude is large compared to the decay length of the tunneling current, the current appears as a series of Gaussian peaks.

rent $I$, and the natural logarithm of $I$ for an ideal exponential current-distance dependence.

Figure 4 shows a simultaneous measurement of topography (constant average current) $\widetilde{\kappa}$, frequency shift $\Delta f$, and damping signal $\Delta E_{c}$ per oscillation cycle. The angle between $\vec{A}$ and the surface normal was $\theta \approx 20^{\circ}$. The cantilever amplitude was $A=100 \mathrm{pm} \pm 4 \mathrm{pm}$. At a single adatom defect site in the right bottom of Fig. 4(c), the frequency shift is positive because of a very small tip-sample distance, where repulsive forces are acting. ${ }^{9,12,13}$ The presence of a single atomic defect proves that the tip has a single front atom and multitip effects can be ruled out. The average damping signal is $\overline{\Delta E_{c}}$ $=170 \mathrm{meV}$ and exceeds the internal damping of the cantilever by $\approx 10 \mathrm{meV}$. On an atomic scale, $\widetilde{\kappa}$ shows a strong variation. The maximum value of $\widetilde{\kappa}$ is $\approx 65 \%$ of the nominal
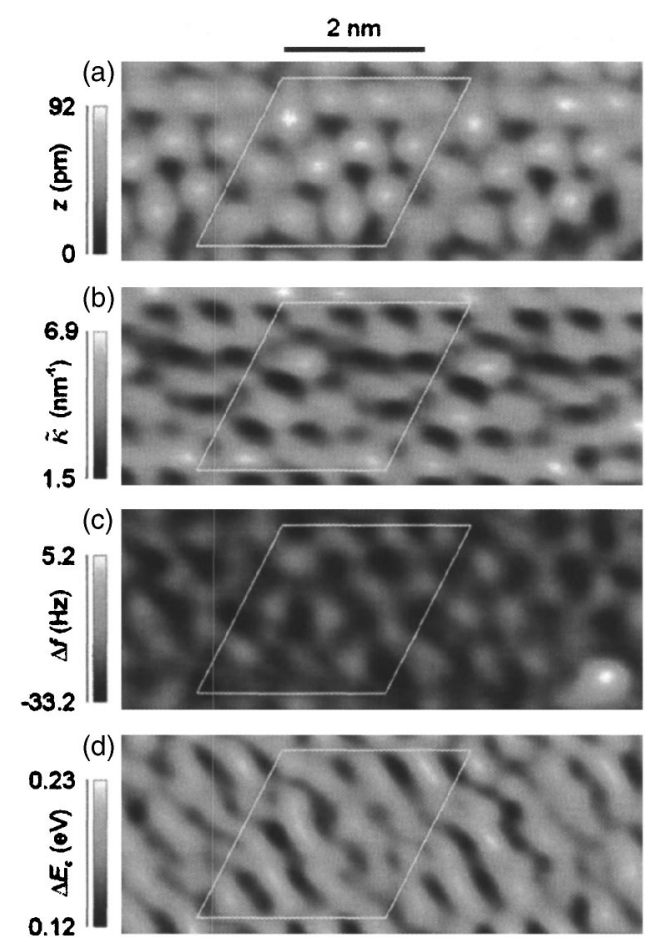

FIG. 4. Simultaneous measurement of topography (constant average tunneling current) (a), $\widetilde{\kappa}$ (b), frequency shift $\Delta f$ (c) and damping signal $\Delta E_{c}$ per oscillation cycle (d). The image was acquired in the dynamic STM mode with a tungsten tip on the silicon $(111)-(7 \times 7)$ surface and a qPlus sensor Ref. 11 with a quality factor of $Q=2240$. The surface unit cell is indicated with white diamonds. Sample bias: $20 \mathrm{~V}$, average tunneling current $I_{\text {av }}$ $=0.8 \mathrm{nA}$, amplitude $A=0.1 \mathrm{~nm}$. 
value of $\kappa$ for this tip-sample system (work-function $\Phi$ $\left.\approx 4.5 \mathrm{eV},{ }^{14}\right)$. The average of $\widetilde{\kappa}$ is less than $50 \%$ of the nominal value of $\kappa$. This lowering of the measured average $\tilde{\kappa}$ can not be explained with the hypothetic geometry factor $\cos (\theta)$ for the case of tunneling between parallel surfaces, which would yield only $6 \%$ of the deviation. However, a considerable lowering and the strong spatial variation are in agreement with earlier measurements using a tungsten tip on a silicon (113) surface. ${ }^{5}$ A lowering was also observed in the first current-distance measurements at a voltage of $60 \mathrm{mV} .^{2}$ The variation of $\tilde{\kappa}$ at a subatomic scale can be explained with geometry effects in the orbital model for the tunneling process $^{3,9,12}$ as shown above. At some adatoms of the silicon $(111)-(7 \times 7)$ unit cell, $\widetilde{\kappa}$ shows a maximum as expected in the simple orbital model. The lateral displacement of the maxima of $\widetilde{\kappa}$ with respect to the topographic maxima is in very good agreement with the results of a model calculation for a $s$ tip on the silicon surface at the experimental conditions $\left(\theta \approx 20^{\circ}\right)$. However, along the surface projection of $\vec{A}$ (vertical direction in the paper plane of Fig. 4), $\widetilde{\kappa}$ decreases faster than in the model, probably caused by displacements of tip- and sample atoms at the small tip-sample distance. The variations in the maximal values of $\widetilde{\kappa}$ between the four types of adatoms on the silicon $(111)-(7 \times 7)$ surface point to variations in the local work function.

Partially, the lowering of the maximum value of $\tilde{\kappa}$ may be caused by the spatial extension of the tip atom or atom cluster. An extended tip results in a blurring of the image, compared to the theoretical images and should result in a lower value of $\widetilde{\kappa}$. In proximity to the sample the polynomial radial factor of the atomic basis functions can additionally cause a lowering of $\widetilde{\kappa}$ compared to $\kappa$. Finally, the finite voltage causes changes in the barrier shape between tip and sample, which might also lower the value of $\tilde{\kappa}$.

The observed features may be a consequence of the structure of the surface wave functions which are probed in dynamic STM. ${ }^{3,9,12}$ However, displacements of tip and sample atoms and the formation of chemical bonds also have to be considered because of the very small tip-sample distance. ${ }^{15}$ Figure 4(c) shows that the frequency shift is less negative on top of the $\mathrm{Si}$ adatoms than between the adatoms. Because adatoms exert strong attractive forces on the tip before contact, ${ }^{16}$ we conclude that the short-range forces acting between tip and sample in Fig. 4 are already repulsive. Bond formation can additionally cause variations in the decay con- stant images on an atomic scale. By which extent the measured variation is caused by mechanical deformations of the tip and/or sample, the formation of a chemical bond or by geometry effects solely because of the structure of the surface electron states can not be determined at this stage.

In conclusion, we have presented simultaneous measurements of the tunneling current, the work function, and the tip-sample forces with atomic resolution. Future improvements of the technique could be achieved by utilizing force sensors with even higher spring constants, such that smaller oscillation amplitudes become possible. Orienting the sensor oscillation exactly perpendicular to the surface would facilitate the interpretation of the data. We suggest that differences in the local barrier heights might help to identify the charge state or species of single atoms. The topographic corrugation can be increased dramatically using higher angular momentum tip states. ${ }^{3,9,12}$ For atomic scale measurements with such tips, an enhanced corrugation of the work function images and higher harmonics of $\ln \left(I / I_{0}\right)$ is expected.

The authors thank S. Hembacher for discussions. This work is supported by the Bundesministerium für Bildung und Forschung (project EKM13N6918).

${ }^{1}$ E. C. Teague, J. Res. Natl. Bur. Stand. 91, 171 (1986).

${ }^{2}$ G. Binnig, H. Rohrer, C. Gerber, and E. Weibel, Appl. Phys. Lett. 40, 178 (1982).

${ }^{3}$ C. J. Chen, Introduction to Scanning Tunneling Microscopy (Oxford University Press, New York, 1993).

${ }^{4}$ G. Binnig, H. Rohrer, C. Gerber, and E. Weibel, Phys. Rev. Lett. 49, 57 (1982).

${ }^{5}$ J. B. Pethica, J. Knall, and J. H. Wilson, Inst. Phys. Conf. Ser. 134, 597 (1993).

${ }^{6}$ R. J. Hamers, R. M. Tromp, and J. E. Demuth, Phys. Rev. Lett. 56, 1972 (1986).

${ }^{7}$ R. Garcia and R. Perez, Surf. Sci. Rep. 47, 197 (2002).

${ }^{8}$ F. J. Giessibl, Rev. Mod. Phys. 75, 949 (2003).

${ }^{9}$ M. Herz, F. J. Giessibl, and J. Mannhart, Phys. Rev. B 68, 045301 (2003).

${ }^{10}$ S. Kitamura and M. Iwatsuki, Appl. Phys. Lett. 72, 3154 (1998).

${ }^{11}$ F. J. Giessibl, Appl. Phys. Lett. 73, 3956 (1998).

${ }^{12}$ M. Herz, Ph.D. thesis, University of Augsburg, Germany (2003).

${ }^{13}$ S. Hembacher, F. J. Giessibl, J. Mannhart, and C. F. Quate, Proc. Natl. Acad. Sci. U.S.A. 100, 12539 (2003).

${ }^{14}$ N. W. Ashcroft and N. D. Mermin, Solid State Physics (Saunders College, Philadelphia, 1981).

${ }^{15}$ W. A. Hofer, A. J. Fisher, R. A. Wolkow, and P. Grutter, Phys. Rev. Lett. 87, $236104(2001)$.

${ }^{16}$ F. J. Giessibl, Science 267, 68 (1995). 